\title{
Filigrane
}

Écoutes psychothérapiques

\section{Si la vie vous intéresse}

\section{Lorraine Boucher}

Volume 21, numéro 2, automne 2012

Psychanalyse et engagement II

URI : https://id.erudit.org/iderudit/1015195ar

DOI : https://doi.org/10.7202/1015195ar

Aller au sommaire du numéro

Éditeur(s)

Revue Santé mentale au Québec

ISSN

1192-1412 (imprimé)

1911-4656 (numérique)

Découvrir la revue

Citer cet article

Boucher, L. (2012). Si la vie vous intéresse. Filigrane, 21(2), 11-26.

https://doi.org/10.7202/1015195ar

\section{Résumé de l'article}

L'auteure explore les particularités de son engagement personnel dans un contexte de consultation psychosociale en protection de l'enfance. Elle cherche à décrire l'engagement collectif ainsi que l'engagement plus personnel d'une psychanalyste. L'exemple clinique d'une jeune enfant sert à illustrer l'ampleur et la nature de l'engagement partagé en situation périlleuse de survie psychique. Se définit peu à peu le souhaitable engagement bien tempéré.
Ce document est protégé par la loi sur le droit d'auteur. L’utilisation des services d’Érudit (y compris la reproduction) est assujettie à sa politique d'utilisation que vous pouvez consulter en ligne.

https://apropos.erudit.org/fr/usagers/politique-dutilisation/ 


\section{Si la vie vous intéresse}

\section{Lorraine Boucher}

L'auteure explore les particularités de son engagement personnel dans un contexte de consultation psychosociale en protection de l'enfance. Elle cherche à décrire l'engagement collectif ainsi que l'engagement plus personnel d'une psychanalyste. L'exemple clinique d'une jeune enfant sert à illustrer l'ampleur et la nature de l'engagement partagé en situation périlleuse de survie psychique. Se définit peu à peu le souhaitable engagement bien tempéré.

' ' 'ai résisté quelques mois à l'appel pourtant bienveillant de la revue Filigrane sur le thème de l'engagement. Je me méfie de l'engagement trop personnalisé. L'engagement authentique ne met-il pas davantage en valeur l'autre que soi-même? S'il se fait l'objet d'une reconnaissance, ne devrait-il pas venir par autrui, après un long parcours? De plus, dans ma pratique analytique, je me méfie comme de la peste de mon propre besoin d'engagement. Un tel besoin peut contaminer mon écoute, m’éloigner du nécessaire respect du choix des autres, compromettre la modestie inhérente au travail de l'ombre du psychanalyste. Il comporte le risque d'instaurer la préséance d'une offre extérieure sur la demande assumée par le sujet en démarche. Je préfere de beaucoup l'engagement partagé, que je peux bien sûr favoriser, comme celui avec l'analysant qui ne me laisserait pas le monopole de l'engagement au sein de sa propre démarche. Ou encore l'engagement partagé avec tous les membres d'une équipe de travail avec laquelle je partage un parti pris de vie physique ${ }^{1}$ et de vie psychique.

Une publicité de l'armée canadienne me sollicite à l'appui de ma réticence à écrire sur mon engagement. À l'arrière-plan, une scène atténuée de guerre aux couleurs de mission de paix en zone de conflit armé, à l'avantplan des scènes d'allure héroïque et puis cette phrase ambiguë: «Si la vie vous intéresse, engagez-vous. » Soit le risque de mort au menu et l'engagement avec la vie trépidante comme argument. Une ordalie: frôlez la mort et vous vous sentirez moins coupable d'être en vie, vous achèterez votre ciel. Ou encore, engagez-vous, vous vous sentirez enfin utile aux yeux des autres. Finies les allusions au confort de l'approche des analystes du divan. Les analystes savent maintenant sauter en parachute, ramper dans les tranchées et se serrer dans les rangs. 
À minima, je n’hésiterais pas à me dire engagée pour la vie psychique et ses pré-conditions d'existence quel que soit l'endroit où je pratique, en bureau privé comme en institution. Dans ce qui suit, j'aimerais toutefois saisir l'occasion qui m'est offerte pour témoigner d'une pratique de consultante, davantage analyste que psychiatre, auprès d'éducateurs et de travailleurs sociaux dans un milieu psychosocial non psychiatrique, non psychanalytique (Boucher, 2004). C'est ce type de milieu qui est tantôt qualifié par René Roussillon de "débarras et d'interstice» (Roussillon, 1987, 165) ou par Michel Soulé de «milieu infra-psychiatrisable» (Soulé et Noël, 1971, 608). Cet engagement atypique depuis plus de 20 ans dans un milieu marqué d'innombrables réorganisations institutionnelles m'offre à l'occasion le spectacle de champs de bataille dévastés et de pertes excessives de troupes stratégiques. La réalité crue des situations de violence, d'abus et de négligence des enfants opacifie ce qui peut être appréhendé de la réalité psychique singulière de l'enfant-sujet. La multiplicité des intervenants aux discours singuliers sur l'enfant crée une distorsion dans la représentation que peut se faire l'analyste de la scène interne du jeune sujet.

Je résiste toutefois à parler d'engagement exceptionnel car où que je sois, je me sens la même. Les deuils que je fais tantôt en institution, tantôt en bureau privé, ne sont pas identiques, mais les défis peuvent se côtoyer. L'institution nous amène à voir et entendre l'insoutenable quelquefois. La nature de la clientèle peut induire un sentiment d'arriver trop tard ou trop tôt avec l'outil psychanalytique, ce dernier exercé d'abord en condition d'atmosphère plus contrôlée pour des conditions psychopathologiques plus évoluées. Quoiqu'il en soit, la même disposition d'ouverture est nécessaire face à ce qui est présenté, avec le souhait peut-être téméraire de maintenir une capacité de rêverie ou d'attention flottante malgré des conditions variables. L'analyste propose son accueil, son écoute, quelques mots potentiellement porteurs, le patient ou le groupe disposent. Pour l'analyste qui connaît de meilleures conditions d'exploration en cabinet privé, l'ampleur des deuils sur le champ de bataille psychosocial constitue le plus gros enjeu. Le deuil le plus significatif est sans contredit celui d'une pratique individuelle en profondeur et de longue haleine. En cela, l'analyste partage les sentiments de toute la communauté engagée et affectée par la carence affective avec son enjeu contre-transférentiel de base «du toujours trop peu et du jamais assez».

Pour vous permettre de mesurer la nature de l'engagement partagé, le mien dans ma tentative d'apporter ma contribution psychanalytique, mais aussi celui des autres membres d'une équipe psychosociale, je vous ferai ren- 
contrer une enfant, Méli-Mélo. Vous constaterez que contrairement au cadre analytique plus classique, l'analyste se fait ici plus discrète auprès de l'enfant dans le contexte d'un suivi délégué, mais elle n'est pas moins soucieuse de l'état psychique de cet enfant et de son entourage. Nous découvrirons peu à peu l'empreinte du maternel créé, sa qualité et son exigence. Il nous servira de fil d'Ariane ${ }^{2}$.

\section{Méli-Mélo: sur la voie d'un maternel de vie à créer}

Méli-Mélo est née d'une mère âgée de 15 ans, le père en avait quatre de plus. Ils se sont rencontrés alors qu'ils étaient tous les deux dans des foyers de groupe de réadaptation. L'enfant connaît deux signalements en protection, le premier à l'âge de quatre mois et l'autre à quatre ans. À quatre mois, bébé est pincée violemment par son père. La petite est non protégée par la mère qui consomme des drogues à cette époque. Des mesures de suivi psychosocial sont ordonnées. Méli-Mélo connaît beaucoup d'instabilité auprès de ce jeune couple suivi étroitement par les services de l'enfance. Le couple se sépare en cours de route pour cause de violence conjugale et de menaces de mort par le père à l'endroit de la mère. À l'âge de deux ans, Méli-Mélo est confiée à la grand-mère maternelle avec l'objectif, dit-on, de stabilisation du milieu de vie, la mère étant très perturbée. Les qualités maternelles de cette grand-mère ne sont pas évaluées en grande profondeur, puisqu'il s'agit d'une parente stable, travaillant comme enseignante, significative pour l'enfant et offrant une assurance de sécurité lors des contacts réguliers avec la mère. Éventuellement, alors que Méli-Mélo a près de quatre ans, la grand-mère, voulant responsabiliser sa fille, demande que celle-ci vive avec la petite en appartement supervisé. C'est peu après, lors d'un deuxième signalement, que Méli-Mélo est retrouvée seule dans la rue, barbouillée de caramel, tandis que sa mère est profondément endormie à la maison. Méli-Mélo est difficilement protégée par la loi tout comme ces enfants si bien décrits par Maurice Berger, «des enfants qu'on sacrifie au nom de la protection de l'enfance» (Berger, 2005).

Tant dans le discours qu'on me tient en réunion d'équipe que dans le dossier de l'enfant, il n'est pas question du vécu intérieur de Méli-Mélo. L'actualité prend le devant de la scène, multipliée par le nombre d'intervenants qui se sont succédé dans la vie de l'enfant ainsi que par l'énoncé des évènements traumatiques. Nous pouvons toutefois retrouver, si on cherche bien, des indices de souffrance précoce bien avant que l'enfant n'ait un Moi plus structuré (Bonneville, 2010): des hurlements sinistres chez un nourrisson qui ne cherche pas de réconfort auprès de sa mère; une histoire d'épisodes d'immobilité, de 
gel, alternant avec des épisodes de grande agitation; certains procédés auto-calmants; une apparente insensibilité à la douleur; des épisodes d'agrippement lors des séparations d'avec la grand-mère; des crises violentes où l'enfant frappe tout ce qui bouge. Un peu plus tard sont identifiés des troubles grandissants du langage tant expressif que réceptif.

Une approche automatique dans ce type de cas amènerait les intervenants sociaux à multiplier les évaluations et ateliers de stimulation, et à se préparer pour la Cour de la jeunesse un an plus tard, experts à l'appui. C'était cette approche qui avait prévalu lors de la fermeture du dossier psychosocial après le premier signalement. Cette fois-ci, le groupe en consultation avec moi cherche moyen d'offrir immédiatement à cette enfant un cadre structurant, favorisant un maternel de meilleure qualité, en attendant que les décisions juridiques se précisent. Cela pourra nous conduire vers une avenue plus propice aux prises de décision ultérieures. Vous constaterez que sur la voie d'un maternel à créer par nous tous, c'est une enfant que nous aurons trouvée. Nous avons pu progressivement faire partager nos découvertes à son entourage qui en devenait témoin, entourage qui fut transformé par cet engagement collectif.

Lorsque Méli-Mélo m’est décrite, à l'âge de cinq ans, elle suscite bien peu d'espoir. Les intervenants me parlent de transmission intergénérationnelle et d'une enfant identique à sa propre mère. Méli-Mélo a le même lit que sa mère lorsque celle-ci était petite, les mêmes jouets, la même attitude tyrannique et obstinée, et elle est maintenant sous les soins de la même mère et du même père. En effet, le juge vient de confier Méli-Mélo pour un an à sa grand-mère maternelle pendant la semaine, et au grand-père maternel, pour la fin de semaine, le couple vivant séparé. Dans un an, une décision juridique de projet de vie définitif sera prise. Est anticipé par le juge un retour de l'enfant à sa mère si cette dernière se reprend, dit-on, en mains.

La mère de Méli-Mélo, s’opposant à tout, dont à la décision du juge, est défiante des services sociaux. Elle n'offre aucune collaboration au suivi psychosocial et psychologique. Des visites supervisées lui sont offertes qu'elle doit confirmer à l'avance, tant elle est instable dans son investissement de la petite. Son attitude lors de ces visites supervisées est dans l'ensemble assez brusque et ses propos peu rassurants, dénigrants et projectifs. Elle arrive quelquefois en retard comme si de rien n'était et part en pleine visite sans avertir l'enfant, simplement parce qu'elle ne se sent pas bien, possiblement lorsque sa fille devient brusque et en recherche de contrôle. La mère semble claustroagoraphobe et refuse toute évaluation ou soin psychiatrique. Nous ne 
pouvons compter sur une alliance avec elle pour le moment, ce qui ne veut pas dire que nous sommes incapables de travailler avec l'enfant ses représentations maternelles.

Notons aussi que pendant ces visites supervisées, Méli-Mélo, anxieuse et peu expressive de sa propre détresse, devient passablement dissociée lorsque sa mère s'agite. Les visites réactivent dans les heures qui suivent un vécu traumatique avec son lot de cauchemars et de crises désorganisées. Des mesures pare-excitantes sont aménagées par la travailleuse sociale afin de limiter les effets perturbateurs sur la petite de ce type d'effraction maternelle. Par exemple, est assurée la présence soutenue d'une éducatrice-accompagnatrice pour l'enfant lors des visites supervisées, devenues moins fréquentes et plus courtes. Nous proposerons à la mère des rencontres intercalées de «retour sur la visite supervisée».

De façon aigüe pour l'équipe en réflexion, des questions se posent : qui est Méli-Mélo? Que pense-t-elle, que comprend-t-elle, qu'a-t-elle imaginé pour combler le vide et le silence malgré l'agitation ambiante? Quand je parle de silence ici, je fais référence au manque de nourriture du moi, un manque de paroles à son adresse pour l'aider à comprendre ce qui lui arrive, du silence qu'elle fait possiblement sien à travers ses troubles de langage. Il est donc décidé qu'une éducatrice ira toutes les semaines jouer avec l'enfant à la résidence de la grand-mère. Elle cherchera à prendre contact avec cette enfant décrite comme un petit monstre sauvage, en retrait, difficile à comprendre tant son discours est limité et ses réactions imprévisibles. Lorsque l'enfant nous parle, elle semble créer un mur de mots effilochés à débit constant, incompréhensibles, comme s'il lui importait moins d'être comprise que de maintenir un agrippement. Cet amoncellement de mots viendrait-il plutôt constituer une barrière protectrice face à l'autre, se demandent ceux de l'équipe qui l'ont rencontrée? Un trouble orthophonique est par ailleurs loin d'être exclu.

Le cadre de rencontres à domicile nous apparaît le seul possible dans un premier temps car la grand-mère ne fait pas de demande pour elle-même et ne voit pas la nécessité d'un accompagnement affectif de l'enfant en externe. Toutefois, elle nous ouvre sa porte car elle s'est aperçu que Méli-Mélo apprécie la visite de l'éducatrice à la maison, cette dernière mandatée à l'origine pour l'évaluation du développement. De plus, la mère, non encore déchue de ses droits de décider des soins de l'enfant, se dit en accord avec une aide psychoéducative pour sa fille puisque cela correspond à son désir de devenir elle-même éducatrice un jour. Nous pouvons voir dans la formulation maternelle l'indice d'une confusion des identités. 
Cette offre de jeu partagé n'est pas typique de l'institution psychosociale où les éducatrices se voient normalement confier des mandats à court terme d'évaluation et de conseils éducatifs aux parents. Ces rencontres de jeu avec une éducatrice supervisées par moi pour plus d'un an furent négociées au niveau administratif en invoquant les défenses particulières du milieu d'accueil, mais aussi comme une opportunité d'ouverture à ne pas manquer. Les rencontres de groupe, les supervisions individuelles de l'éducatrice et de la travailleuse sociale, ainsi que les rencontres régulières avec les grands-parents me permettront de faire un résumé du suivi d'un an de Méli-Mélo. J’essaierai de me centrer sur le mouvement illustrant la modification des représentations maternelles chez l'enfant. Ces représentations maternelles ne sont pas, pour l'analyste, superposables aux représentations de la mère. Vous y apercevrez l'ampleur d'un engagement partagé mais aussi sa nature.

\section{Un engagement de jeu partagé avec l'enfant et les grands-parents}

Dès le début, au cours des visites chez la grand-mère, une impression générale se précise. Cette impression est ressentie tant par l'éducatrice que par la travailleuse sociale; elle est corroborée par des phrases dites secrètement par Méli-Mélo. En effet, un silence malsain et de lourds secrets sur les difficultés de la mère entourent les tensions familiales pourtant omniprésentes. Méli-Mélo enfreint le silence pour nous contraindre à le respecter tout aussitôt: «Dis-lui pas à ma maman que je la trouve méchante parce qu'elle va être fâchée contre moi. Maman fait mal à mon cœur, j'ai voulu appeler la police. » Nous sentons par ailleurs la grand-mère affairée aux soins primaires, incapable d'un accompagnement affectif, figée dans son propre silence et probablement sa propre douleur. Des fantaisies de princesse stationnée dans un palais de glace frayent dans les associations de l'équipe. La solitude de cette enfant nous inquiète impérativement. Les rencontres régulières de jeu avec l'éducatrice sont présentées à l'enfant comme une possibilité de jouer avec quelqu'un «à ce qui fait mal à son cœur ou à ce qui lui fait du bien».

Une première rencontre avec les grands-parents a pour but de répondre à leurs désirs de discuter avec nous de l'évaluation psychologique récente de Méli-mélo et de l'indication d'un accompagnement spécialisé (QI moyen, insécurité avec l'adulte, trouble relationnel majeur mère-enfant, pas de trouble de l'attention mais trouble anxieux, pas d'évidence de trouble majeur de langage). Les grands-parents se demandent si les crises faites aux moments des transitions ou des séparations vont enfin s'amender. Nous explorons les 
défis que Méli-Mélo traverse et son besoin d'accompagnement affectif quotidien. Nous identifions la difficulté des grands-parents à s'investir pleinement comme parents substituts de Méli-Mélo, plutôt que comme parents en attente indéfinie du retour de leur fille à ses responsabilités. Ils entraînent ainsi leur petite fille avec eux sur cette voie de garage et surtout d'enfermement affectif.

Nous regardons aussi quelques dessins qu'ils nous ont amenés : une maison noire mélancolique représentant celle de grand-papa; un coloriage pour la grand-mère d'un cheval à la tête, au corps et aux membres de couleurs différentes, comme un cheval rapiécé en quête d'identité; enfin, une fleur rose, en provenance de l'école, bien constituée mais perdue au milieu d'un champ gris au ciel sombre. Les dessins offerts à l'éducatrice foisonnent d'un minuscule personnage allumette aux doigts démesurément grands, personnage sans visage à part de grands yeux. Déjà dans ces dessins nous pouvons pressentir l'indice d'un moi-allumette clivé, quasi atomisé, un accrochage au percept avec des yeux plus grands que la panse, une lutte importante contre l'avidité du minuscule personnage, ainsi qu'une expression personnelle teintée par la nature de la personne qui reçoit le dessin. En effet, le grand-père nous était apparu mélancolique, la grand-mère bien conformiste et opératoire, l'éducatrice grandement expérimentée et invitante, tandis que l'école, empathique à Méli-Mélo, avait reconnu d'emblée ses difficultés de socialisation. La représentation qu'offre Méli-Mélo d'elle-même est instable et semble tributaire de l'environnement relationnel ambiant.

Les premières rencontres avec l'éducatrice permettent un apprivoisement de l'enfant, manifestement en attente de jouer. Les scénarios qu'elle aborde sont chargés de violence. Il s'agit de scènes macabres d'enfants dépecés, jetés à la rue, négligés, invectivés brutalement par un personnage adulte que MéliMélo joue froidement avec ses poupées ou en demandant à l'éducatrice de jouer l'enfant. L'imago maternelle sauvage est au rendez-vous. L'éducatrice se demande si Méli-Mélo ne fait que se transposer elle-même répétitivement et s'il y un jeu véritable. Elle se demande si l'enfant s'amuse vraiment. L'éducatrice craint l'impasse et la stagnation dans un scénario sado-masochique en externalité qui n'amène pas d'élaboration psychique mais plutôt une décharge. Approcher son monde interne est encore trop exigeant. Nous pouvons faire l'hypothèse que Méli-Mélo n'est pas encore capable d'élaborer un fantasme de fustigation structurant (Boucher, 2000). L'érotisation de la scène traumatique n'est pas encore possible, Méli-Mélo a donc recours à des procédés d'autorégulation par décharges " auto-excitatoires », ce que son entourage appelle des crises. La fin des rencontres est très difficile, l'enfant ne veut 
pas cesser. Il y a des crises au cours desquelles Méli-Mélo crie et frappe la grand-mère qui s'approche pour reprendre contact au départ de l'éducatrice. La grand-mère est sans voix, désemparée.

Une séance développe le thème de l'enfant omnipotent: «Moi, je suis capable toute seule, j'ai 11 ans. (Elle se coiffe.) Je peux faire ma couette toute seule même si ma maman ne veut pas. » Méli-Mélo joue alors un personnage autoritaire, intransigeant et tyrannique, qui sourit lorsqu'il impose des règles démesurées au jeune garçon joué par l'éducatrice. Méli-Mélo arrive difficilement à contrôler ses pulsions agressives et s'emporte sadiquement avec sa partenaire de (non-) jeu. Le cadre doit être rappelé à l'enfant. Ensuite, deux amies jouent ensemble mais l'une d'entre elles fait sans cesse obstruction à tout ce que propose l'autre. L'éducatrice lui demande, sur un ton qu'on imagine empathique, si elle s'amuse vraiment à ce jeu-là? Cette question aura pour effet d'ouvrir sur ce qu'elle n'aime pas quand sa maman s'en va et qu'elle lui dit qu'elle est assez grande maintenant. Nous voyons là la reprise du thème d'un moi idéal omnipotent, avec ce « je suis capable tout seule » et aussi cette puissante opposition qu'elle fait sienne dans une sorte de contrainte identificatoire à l'agresseur (Roussillon, 2005).

Méli-Mélo, plus calme, continue avec un autre jeu. Elle met en scène cette fois un garçon qui fait des cauchemars. L'éducatrice, telle une mère bienveillante, s'approche. Elle doit se coucher à côté de l'enfant qui a peur sans dire un mot. Méli-Mélo commente subitement: "J'aime pas ça être seule.» L'éducatrice-mère se couche à côté mais le cauchemar ne lui sera pas raconté. Méli-Mélo vient de faire la différence entre être seule et n'être pas seule. Elle n'aime pas ça être seule, mais elle n'est pas encore prête à nous faire part du rêve. L'objet d'attachement est investi, mais l'objet psychique est encore craint car il porte encore une trop grande charge traumatique.

À l'annonce des cinq minutes qui restent à la séance, Méli-Mélo devient subitement autosuffisante dans une activité d'assemblage. Elle recherche le regard admiratif de l'éducatrice sur ce qu'elle sait faire maintenant. C'est sa manière d'annoncer qu'il n'y aura peut-être pas de crise cette fois. Tel que discuté auparavant avec elle, la grand-mère est invitée à cesser ses activités et à venir se joindre pour quelques minutes pendant la transition. La grandmère commente qu'elle est surprise; bien qu'elle ne voulait pas entendre, elle a entendu: sa petite fille revient sur des thèmes douloureux. Elle ne savait pas qu'une enfant pouvait vivre des choses avec autant d'intensité et qu'elle veuille même jouer à ça. La grand-mère constate que ce sont les mêmes jeux auxquels Méli-Mélo s'adonne parfois lorsqu'elle se retrouve seule. 
Peu à peu, ce sera Méli-Mélo qui ira d'elle-même chercher la grand-mère pour les dernières minutes avant le départ de l'éducatrice. Les jeux de société apparaissent dans la relation avec la grand-mère, le paquet voleur et les cassetêtes.

Reviendra ensuite pour disparaitre progressivement ce jeu récurrent : un bébé est jeté contre un mur, soumis à l'hostilité d'un adulte insensible. «Estce qu'il pleure ce bébé? » "Non, ce petit frère ne pleure pas. Aucun bébé ne pleure dans cette famille. C'est une famille comme cela. » « Et pourquoi on le jette le bébé?» «Parce que son papa est mort et que le bébé ne devait pas pleurer. Jamais plus il ne pleurera, le bébé.» Manifestement, Méli-Mélo se débat encore avec une imago maternelle terrifiante, fermée à l'intériorité de l'enfant et à la triangulation, mais le papa fait quand même son entrée par la porte de la mort.

Une séance sera particulièrement éprouvante, l'éducatrice ayant été retardée, alors qu'à la visite supervisée précédente, la mère ne s'était pas présentée. Méli-Mélo qui feint d'abord d'ignorer son arrivée tardive, semble jouir de mettre l'éducatrice en position humiliante et va jusqu'à lui donner un coup de pied. La séance est alors écourtée, comme convenu lorsque l'enfant agit violemment sans faire semblant. Sur une autre scène, les visites supervisées de la mère sont renégociées à la lumière de son absentéisme. La situation est très difficile pour l'éducatrice qui sent l'enfant plus seule que jamais. Elle craint que la thérapie ne serve qu'à compenser un milieu qui n'accompagne pas véritablement l'enfant lorsqu'elle essuie des blessures et des abandons véritables. Les grands-parents ne disent pas un mot lorsque la mère s'absente au rendez-vous prévu. L'éducatrice aimerait sentir moins de déni ambiant. De son côté, tel que convenu en supervision, elle veut revenir sur son propre retard à la séance de jeu.

À la séance suivante, Méli-Mélo aborde la difficulté de ne pouvoir dire à sa grand-mère qu'elle hait sa propre mère. D'autant que cette grand-mère se fait un honneur de lui dire: «Ta mère est fâchée contre moi, mais moi je ne suis pas fâchée contre ta mère.» Cette offre identificatoire ainsi que cet interdit de ressentir un affect désagréable empêchent toute expression plus secondarisée chez Méli-Mélo. Alors que nous lui offrons une parole plus libre, cet interdit venant de sa grand-mère a un effet mélangeant pour elle. Le terme méli-mélo est alors introduit par l'éducatrice pour la première fois.

Nous organisons un rendez-vous avec les grands-parents en présence de l'éducatrice et de la travailleuse sociale afin d'explorer l'enjeu de l'accompagnement affectif au quotidien. Nous y remarquons que l'intervention de 
l'éducatrice a un effet d'étayage pour la grand-mère, à l'insu même de l'éducatrice qui s'en voit surprise. La grand-mère dit maintenant tendre à se faire disponible à Méli-Mélo lors d'évènements malheureux mais elle s'avoue plus limitée que l'éducatrice. Jamais, dit la grand-mère, elle n'irait se réfugier sous une table transformée en grotte comme le fait l'éducatrice dans le jeu, ou jamais elle n'irait balancer l'enfant comme un sac de vidanges quand le demande Méli-Mélo. Toutefois, elle raconte souriante qu'elle essaie de faire comme l'éducatrice à sa manière: avant le coucher, elle raconte à l'enfant des contes de fées en prenant des voix tant humaines qu'inhumaines.

Par ailleurs, les nouvelles de l'école sont bonnes: Méli-Mélo n’est plus considérée un cas difficile, elle s'est fait des amies et elle n'a que quelques rendez-vous en orthophonie. Nous abordons enfin avec les grands-parents le problème qui les inquiète encore : les crises de Méli-Mélo. Nous cherchons à dégager avec eux le sens possible de ces crises qui apparaissent, pour la plupart, en contexte d'abandon ou évocateur d'abandon. Il leur est donc suggéré d'introduire activement avec leur petite-fille, dès que cela se produit ou s'annonce, le difficile sujet des absences ou des retards. L'exemple des absencesabandons de la mère de Méli-Mélo leur est offert, ainsi que la suggestion qu'ils initient eux-mêmes la parole autour de cette réalité pénible mais incontournable.

Comme vous pouvez le constater, nous avons d'abord respecté la défense grand-maternelle à reconnaître ses difficultés à échanger des mots signifiants pour l'enfant sur les absences de sa mère. Toutefois, le passage par les préoccupations grands-parentales concernant les crises nous a permis d'unir les grands-parents autour d'une compréhension plus pertinente à leurs yeux. L'évolution nous laisse entrevoir qu'ils ont su profiter de cette exploration au bénéfice de Méli-Mélo.

Aussi, le jeu dans les semaines qui suivent aborde des scènes de conflits entre amies et entre sœurs. Il y a beaucoup de conflits et peu de réconciliations. Il y a des moments de confusion, comme si Méli-Mélo perdait pied entre la réalité et la fiction. Le thème de l'abandon d'enfant par le parent odieux a laissé place au thème de l'enfant abandonnée par ses amies parce qu'elle ne se comportait pas bien. Les adultes sont un peu plus périphériques, et surtout moins dangereux. Les conflits fraternels ne sont pas réglés. La représentation maternelle s'adoucit, mais elle n'est pas encore un soutien de l'estime de soi puisque l'enfant odieux engendre l'abandon qu'il subit. MéliMélo reste très dépendante du monde externe pour son économie narcissique. 
Méli-Mélo a développé enfin un rituel de sortie de séance. Le rituel est: « Je te donne un câlin, ou je ne t'en donne pas.» Tout dépend de mille et une raisons.

Un saut qualitatif est enfin perceptible au bout de quelques mois, associé à un plus grand confort au départ de l'éducatrice. Méli-Mélo cherche alors activement sa grand-mère qui à son tour lui offre une place bien à elle. Dans son discours spontané, la grand-mère semble autoriser Méli-Mélo à se différencier de sa propre mère. Dans son milieu de vie plus personnalisé maintenant, nous constatons que Méli-Mélo a choisi de nouvelles couleurs pour sa chambre.

Une princesse arrive enfin dans les histoires, l'éducatrice est une alliée, une amie dans le combat contre une sorcière démentielle. Méli-Mélo a des moments de grande frayeur dans le jeu, et aussi beaucoup d'excitation. Elle demande à l'éducatrice d'aller toute seule au combat rencontrer la sorcière qui dit: «Hum, ça sent bon les enfants. » Le saut qualitatif que nous identifions s'associe à l'apparition d'histoires qui ont enfin un début, un milieu et une fin. Peu avant la fin de la séance, Méli-Mélo s’amuse à débuter une nouvelle histoire, donnant à l'éducatrice l'impression qu'elle ne va pas finir. Mais cette histoire est courte et finit en beauté : le prince charmant donne un baiser à celle qu'une sorcière avait figé en statue de sel. Sortir de la séance se fait maintenant de façon active et s'est transformé en plaisir. L'éducatrice princecharmant sort la princesse sur ses épaules après l'avoir sortie du mauvais sort par un, deux, ou trois baisers. Lovée sur l'épaule salvatrice, la jeune princesse de dire: «Te rappelles-tu quand je criais?»

\section{Sur la trace des représentations maternelles}

Même si le cadre thérapeutique était bien loin d'une salle de thérapie de jeu et de son atmosphère plus contrôlée, nous avons pu suivre et soutenir l'évolution des représentations maternelles chez Méli-Mélo. La représentation qu'elle en donnait d'abord était inhumaine et terrifiante; elle prend maintenant le visage d'une sorcière, certes sadique, mais qui aime les enfants pour les manger. La représentation paternelle séparatrice et salvatrice fait son apparition. L'offre d'un soutien maternel élargi, dont je fais partie par mes supervisions, a fait son œuvre et a servi d'étayage à l'entourage psychosocial et à celui plus proximal de l'enfant. Les bagarres de psychologues experts en cour de la jeunesse ne semblent pas être au programme cette fois. La grand-mère comprend l'indication d'une psychothérapie éventuelle pour Méli-Mélo et n'y fera pas obstacle. Elle envisage pour elle-même une démarche de ce type. Plutôt que d'être instrumentalisés, les experts reprennent leurs fonctions 
pertinentes. Ils pourront orienter les intervenants vers une meilleure compréhension de l'enfant et de ses parents. L'indication de psychothérapie sera une évidence alors qu'un an plus tôt elle eut été difficile à réaliser.

Méli-Mélo, actrice et agente de son propre destin, peut maintenant nous parler, plutôt que sans cesse réagir, même si les magasins sont encore des «gamasins». Plusieurs personnes autour d'elle arrivent à la comprendre sans qu'eux-mêmes ne soient sous l'emprise de l'agir.

\section{La vie psychique menacée}

Enfin, l'intérêt que cette équipe manifestement engagée a porté à l'offre d'un maternel vivant en toute urgence s'est avéré crucial pour que Méli-Mélo fasse partie des décisions, pour qu'un sujet advienne. Nous n'avons pas attendu que tout soit stable autour d'elle avant de lui offrir une écoute sensible, soutenue et régulière à travers son potentiel de jeu. Nous nous sommes engagés sur cette voie exigeante pour tous, la participation de la petite et de son entourage immédiat a suivi. Cette écoute active, offerte face à l'urgence d'un support maternel élargi, dans des conditions à saisir et à créer, nous a permis une issue qu'on croyait inespérée un an plus tôt. Là s'exerce ma part d'engagement, s'il en est: qu'en terrain dévasté, l'enfant reçoive une écoute de toute urgence, un tant soit peu analytique, en amont de ce qui sera plus tard une psychothérapie en milieu protégé. Cette écoute demande une analyse tout aussi sophistiquée qu'en bureau privé, à travers la distorsion qu'induit la pluralité des discours sur l'enfant et une implication de l'analyste toujours à négocier cas par cas. Cette écoute reconnaît que l'existence même de la vie psychique peut être menacée dans des conditions de protection psychosociale et juridique manifestes.

Fascinant pour moi demeure ce type de voyage guidé (Boucher, 2007) que nous offre Méli-Mélo quand elle revisite sa propre histoire et sa symptomatologie précoce de nourrisson. Dans ses jeux, les hurlements du nourrisson ont pris la voix des cris et de l'agitation en fin de séance qu'elle évoquera plus tard par son: « Te rappelles-tu quand je criais. » Le gel et la dissociation se sont retrouvés sous le couvert de la princesse figée en statue de sel. L'insensibilité à la douleur s'est dite nécessaire pour ce bébé qui ne pleure pas: «C'est comme cela dans cette famille-là. » L'agrippement de l'enfant plus que l'attachement sain a fait son nid dans les doigts de la fille allumette aux yeux démesurés. Les troubles du langage se sont montrés sous le thème de l'enfant sans bouche et qui plus tard avoue sa peur sans toutefois dire un mot de son cauchemar, même à une mère bienveillante. 
L'engagement du psychanalyste auprès de l'équipe en est un qui tente de faire une place à cette nécessaire élaboration symbolique de ce qui autrement ne serait que symptômes tant de l'enfant que de ceux qui gravitent autour, symptômes toujours en quête de sens. Nous avons vu le silence contagieux, d'une famille à l'enfant et ensuite au sein des discussions introductives sur l'enfant.

L'accompagnement de l'éducatrice et de l'équipe, une invitation à jouer où les gratifications et les contacts charnels restent encore très présents, la fiabilité grandissante du milieu de vie, auront permis à Méli-Mélo une symbolisation grandissante du maternel, même si encore précaire. On voit ici que l'apport maternel, tantôt élargi par notre offre, tantôt plus intime auprès de ses grands-parents, a exercé son rôle non pas palliatif mais structurant. Il a trouvé ses fruits véritables dans un jeu de représentations symboliques maintenant au service de la subjectivité de l'enfant.

Avec une enfant qui a connu un départ si difficile dans la vie, c'est le champ maternel, le sous-bassement symbolique précoce, que nous souhaitions retisser d'urgence pour qu'une princesse advienne. Ce champ précieux n'est en aucun moment un acquis de l'offre d'une présence physique, même dite engagée. Il demande une mère-environnement capable d'un minimum de sensibilité, d'écoute, de dialogue émotionnel avec l'enfant tant au niveau verbal que non verbal. Il nécessite aussi une grande préoccupation quant aux conditions de vie psychique de l'enfant fragile afin de lui offrir le niveau d'excitation ou de pare-excitation optimal.

Emmanuelle Bonneville, psychologue clinicienne dans le Service de pédopsychiatrie du $\mathrm{D}^{\mathrm{r}}$ Maurice Berger, décrit trois phases dans la réanimation psychique de ces enfants précocement traumatisés (Bonneville, 2010). La première phase offre des soins intensifs, des soins primaires mais sensibles. Ces soins réaniment l'espoir de vie chez l'enfant. La deuxième phase rencontre le besoin fusionnel qui risque d'être interminable; l'enfant s'accapare la personne qui vient de le remettre au monde affectivement, mais il le fait sur le mode de l'addiction. L'enfant fait des crises d'accrochage panique, est avide, jaloux, réclame de façon excessive l'exclusivité de l'attention. L'engagement d'une personne trop engagée auprès de l'enfant peut venir en écho à cet accrochage défensif à la sensorialité, participant ainsi au contre-investissement du monde symbolique pressenti périlleux. Il nous faut être attentif à ce que l'engagement de ce super-objet d'investissement affectif ne vienne empêcher une évolution favorable au niveau psychique plutôt que le maintien d'une relation concrète, fusionnelle et interminable. C'est ce que nous avons 
tenté de négocier avec Méli-Mélo en lui proposant un lien affectif générateur d'une aire transitionnelle de jeu afin de favoriser l'élaboration d'un lien plus symbolique à l'objet. Une évolution de la représentation maternelle, passant de l'imago maternelle terrifiante (pré-représentation) à une représentation moins inquiétante, fut d'abord nécessaire avant que ne puisse s'effectuer le saut vers un lien plus symbolique, avec l'animation des contenus psychiques que cela implique. L'élaboration d'une représentation maternelle plus humaine vient atténuer l'angoisse associée au recours à l'objet symbolique. Ce combat à tenir contre une fusion régressive à un seul objet d'investissement concret s'observe à la fin des rencontres : l'éducatrice accompagne MéliMélo dans le transfert proposé de son investissement vers la grand-mère. Par la suite, nous voyons Méli-Mélo devenir plus active, par étayage, pour enfin devenir capable d'amortir ces sauts périlleux par des histoires de fin de séance, témoignant d'une vie symbolique plus riche.

Cette troisième phase décrite par Bonneville est cruciale pour que l'évolution psychique soit favorable; elle exige ce qu'elle appelle la rupture et l'arrachement. Cette phase est impossible sans qu'un objet d'investissement affectif n'offre un sens à l'enfant à partir des indices externes de son agitation interne. Certains parleraient de l'offre d'une réflexivité structurante et d'un affect partagé. Là aussi, l'engagement bien tempéré en est un qui n'offre pas trop vite un sens, mais va plutôt à la rencontre de ce que l'enfant agité peut exprimer de sa propre agitation interne. L'objet secourable offre un sens sur un mode le moins aliénant possible afin de favoriser une métabolisation subjective progressive des événements traumatiques, pour qu'un véritable processus d'après-coup puisse s'effectuer (Boucher, 2007). Aussi, Méli-Mélo, dans ses rencontres d'abord tumultueuses avec l'éducatrice, devient progressivement plus contenue, capable d'une narrativité nouvelle, plus personnelle et plus créatrice. L'apparition d'un passé, d'un présent et d'un futur dans ses histoires incarnées dans les jeux, nous annonce qu'un temps nouveau vient d'arriver.

Nous avons parallèlement remarqué une évolution chez la grand-mère sans que nous ayons travaillé de façon aussi étroite avec elle. Elle s'est sentie progressivement dégagée de ce qui aurait pu devenir la demande d'un lien fusionnel interminable de la part de Méli-Mélo. Sa propre demande relationnelle, restée plus réservée avec nous, était peut-être de même nature que celle de Méli-Mélo. L'éducatrice a néanmoins offert à cette grand-mère, dans son propre milieu de vie, une figure d'étayage plus triangulée, lui permettant une sortie de l'isolement qu'elle ignorait être le sien. 
Nous n'avons pu rejoindre la mère, longtemps sans domicile fixe et toujours très imprévisible. Le père, d'abord fidèle aux visites supervisées, s'est fait inconsistant par la suite. Nous aurions souhaité pouvoir les amener avec nous. Nous avons dû plutôt limiter les effets désorganisants de leur instabilité effective et affective sur Méli-Mélo, ce qui a permis, à elle et à son environnement d'accueil immédiat, une plus grande disponibilité au changement psychique.

Vous comprendrez qu'il nous faut être prudent dans ce genre d'aventure. Il n'est pas possible dans tous les cas. Ici les conditions d'ouverture suffisante semblaient présentes. Il faut aussi s'assurer que l'engagement de l'éducatrice sera significatif en termes de durée et de supervision pour qu'il n'y ait pas d'abandon tragique, d'interprétation sauvage ou d'engagement excessif. L'encadrement intelligent et sensible du milieu psychosocial est crucial. La loi de la protection de l'enfance étant une loi d'exception, l'aventure d'accompagnement et d'engagement de l'analyste implique une fin inévitable, qui sera inévitablement plus hâtive que s'il était le psychothérapeute en cabinet privé. D'une certaine manière, notre engagement sera tantôt qualifié de trop long par certains et de trop court par d'autres. L'analyste n'est pas à l'abri d'une redéfinition perpétuelle de son engagement dans ce contexte de vie psychique menacée. Jusqu'où faut-il mener la vie si elle nous intéresse? Ce pourrait être notre prochaine question.

Avec ce cas clinique, j'ai cherché à vous faire entrevoir ce qui en est d'un engagement qui souhaite être bien tempéré (Donnet, 1995). Il se veut impérativement soucieux du maintien de la vie symbolique alors même qu'il côtoie un univers propice à la mort psychique, tant les traumas, les silences de mort, les dénis ambiants font obstacle à toute élaboration. Nul doute qu'un cas comme celui de Méli-Mélo a nécessité l'engagement actif et sensible d'une éducatrice accompagnée et accompagnante ainsi que d'une équipe attentive. Pour reprendre l'offre de l'armée canadienne, le plus gros défi ne fut pas seulement d'offrir en zone dévastée, voire dangereuse, une présence volontaire et engagée. Le défi fut aussi que cette présence soit équipée pour la guerre mais non belliqueuse, accueillante et génératrice de vie.

Lorraine Boucher 4000 avenue Marcil Montréal. P.Q. H4A 2Z6 lorboucher@sympatico.ca 


\section{Notes}

1. Ceci est inhérent au travail de consultant en contexte de protection de l'enfance, la sécurité physique et le développement de l'enfant étant compromis.

2. Ce cas fut présenté de façon plus succincte dans un atelier théorico-clinique au Congrès des langues françaises de 2011 qui portait sur le Maternel. Bernard Golse animait cet atelier sur l'empreinte du maternel dans la clinique du nourrisson.

\section{Références}

BERGER, M., 2005, Ces enfants qu'on sacrifie... au nom de la protection de l'enfance, Paris, Dunod.

BONNEVILLE, E., 2010, Effets des traumatismes relationnels précoces chez l'enfant, Psychiatrie de l'enfant, 53 (1), 31-70.

BOUCHER, L., 2000, Un parent est battu, Filigrane, 9 (2), 35-53.

BOUCHER, L., 2004, Psychanalyste sans frontière, Psychanalyste en institution et contretransfert, Filigrane, 13 (1), 34-45.

BOUCHER, L., 2007, De la morsure à l'entomologie, Topique, 99, 97-106.

BOUCHER, L., 2007, Paroles soufflées à l'hélium, emmenez-moi sur terre. Paroles bétonnées, emmenez-moi au ciel, Revue française de psychanalyse, 5, 1645-1654.

ROUSSILLON, R., 1987, Espaces et pratiques institutionnelles. Le débarras et l'interstice, in Kaës, R. (dir.), L'institution et les institutions, études psychanalytiques, Paris, Dunod, 221238.

ROUSSILLON, R., 2005, Les situations extrêmes et la clinique de la survivance psychique, in Furtos, J. (dir.), La santé mentale en actes/De la clinique au politique, Toulouse, Éres, 221238.

SOULÉ, M. et NOËL, J., 1971, Le grand renfermement des enfants dits «cas sociaux» ou Malaise dans la bienfaisance, Psychiatrie de l'enfant, 14 (2), 577-620. 